\title{
Propriedades Reológicas e Dinâmicas de Composições Não-Vulcanizadas de Borracha Natural com Celulose Regenerada
}

\author{
Agnes F. Martins, Leila L.Y. Visconte, Regina C.R. Nunes \\ Instituto de Macromoléculas Professora Eloisa Mano, UFRJ
}

Resumo: Neste trabalho, composições não-vulcanizadas de borracha natural (NR) com celulose regenerada (Cel II) foram preparadas por co-coagulação de misturas de látex natural e xantato de celulose. Foi estudada a influência da carga celulósica nas propriedades reológicas e dinâmicas dessas composições, para teores entre 0 e 30phr. As propriedades reológicas avaliadas foram os torques, elástico ( $\mathrm{S}^{\prime}$ ) e viscoso (S"), a tan delta e a viscosidade dinâmica real ( $\left.\eta^{\prime}\right)$. Para estes ensaios foram feitas varreduras de frequiência e arco, à temperatura constante. A viscosidade e a relaxação Mooney também foram estudadas. Os testes dinâmicos foram realizados à frequiência constante, sendo a tangente de perda e o módulo elástico (E') os parâmetros considerados. Os resultados mostraram uma excelente dispersão da carga celulósica na matriz borrachosa, e sua grande influência nas propriedades viscoelásticas da borracha natural sem, no entanto, modificar o perfil do comportamento reológico desta borracha.

Palavras-chave: Borracha natural, celulose regenerada, propriedades dinâmicas, propriedades reológicas.

\section{Rheological and dynamic properties of unvulcanized natural rubber and regenerated cellulose compositions}

Abstract: In this work, unvulcanized natural rubber (NR) and regenerated cellulose (Cel II) compositions were prepared by co-coagulation of natural rubber latex and cellulose xanthate mixtures. The cellulosic filler was added up to $30 \mathrm{phr}$ content. The rheological properties evaluated were the elastic (S') and viscous (S") torques, tan delta, and the real dynamic viscosity ( $\eta$ '). These experiments were carried out at constant temperature with frequency and strain sweeps. Mooney viscosity and relaxation were also studied. The dynamic tests were taken at constant frequency, and the loss tangent and the elastic modulus (E') were considered. The results showed a good dispersion of the cellulosic filler in the rubber matrix and the large influence it imparted on rheological and dynamic properties of natural rubber without changing the rheological profile of this rubber.

Keywords: Natural rubber, regenerated cellulose, dynamic properties, rheological properties.

\section{Introdução}

Fibras curtas, descontínuas, são usadas para reforço de elastômeros com o objetivo de melhorar ou modificar as propriedades termo-mecânicas da matriz, para aplicações específicas ou reduzir custo do artefato final. Uma vez que as fibras podem ser incorporadas ao elastômero juntamente com os outros aditivos, os compósitos resultantes são obtidos em equipamentos convencionais para processamento de borrachas, tais como extrusora ou calandra, e moldados por diferentes tipos de processos, como: compressão, injeção ou transferência. Fibras de celulose são empregadas para reforço de elastômeros, e são escolhidas quando compostos claros são desejáveis ${ }^{[1]}$.

Um outro produto do tipo fibra-elastômero tem origem na mistura de látex de borracha com soluções de celulose, seguida de coagulação do sistema ${ }^{[2,3]}$. Há

Autor para correspondência: Regina C.R. Nunes, Instituto de Macromoléculas Professora Eloisa Mano, UFRJ, Caixa Postal 68525, CEP: 21945-970, Rio de Janeiro, RJ.E-mail:rcnunes@ima.ufrj.br 
mais de $60 \operatorname{anos}^{[2]}$ fibras de celulose foram modificadas por esse processo, com o emprego de soluções de xantato de celulose.

Por analogia à literatura ${ }^{[4]}$, que utiliza xanteto de amido, a co-precipitação de xantato de celulose com vários látices de borracha tem dois aspectos de interesse tecnológico. Primeiro, a co-precipitação, mesmo com baixos teores de xantato, gerando borracha em pó com tamanho de partícula relativamente uniforme, que pode ser facilmente processada utilizando tecnologia dos termoplásticos. Segundo, a celulose resultante atuando como agente reforçador, elevando as propriedades dos vulcanizados para aplicações industriais.

Composições elastoméricas, antes e após vulcanização, têm comportamento intermediário entre o viscoso e o elástico, apresentando propriedades viscoelásticas. O conhecimento do perfil viscoelástico é muito importante para a previsão do processamento, bem como das propriedades físicas dos elastômeros vulcanizados ${ }^{[5]}$.

Os numerosos métodos usados para determinar as propriedades reológicas dos materiais podem ser divididos em duas categorias. Em uma delas, o material é sujeito à tensão constante, e é medida sua deformação, ou taxa de deformação. Ou então, o material é sujeito à deformação constante, ou taxa de deformação, e é medida a tensão resultante. Outra categoria dos testes reológicos é a dependência temporal da viscosidade e do módulo a estímulo de tensão ou deformação constante ${ }^{[6]}$.

Um método mais recente é o teste dinâmicomecânico ou oscilatório, onde as deformações oscilatórias permitem medir, separadamente, os elementos elástico e viscoso de um material viscoelástico. Como este tipo de teste pode ser usado para uma grande variedade de materiais, é considerado um método reológico universal ${ }^{[6]}$.

Neste trabalho, foram estudadas as propriedades reológicas e dinâmicas de composições nãovulcanizadas de borracha natural com celulose regenerada, para teores de carga entre 0 e 30phr.

\section{Experimental}

A co-coagulação das misturas NR-viscose foi feita pela adição, sob agitação, da mistura de látex natural/ xantato de celulose à solução ácida equimolar de ácido sulfúrico e sulfato de zinco. Para melhor homogenei- zação a viscosidade do sistema foi mantida constante, em todas as composições empregadas, pela mesma relação xantato/água. Além disso, o látex natural foi diluído na proporção 2:1. O teor de celulose regenerada variou entre 0 e 30phr. Após a coagulação, as finas partículas de borracha-celulose obtidas foram submetidas à remoção da acidez residual, com água destilada. O produto separado da suspensão aquosa por filtração foi, então, seco em estufa com circulação de ar, a $50^{\circ} \mathrm{C}$, e submetido aos diferentes ensaios.

A viscosidade e a relaxação Mooney foram determinadas em viscosímetro Mooney, da marca Monsanto, modelo MV2000, utilizando rotor pequeno, MS. O ensaio foi realizado de acordo com a norma ${ }^{[7 a]}$ ASTM D 1646.

As propriedades reológicas das composições nãovulcanizadas foram feitas no analisador de processamento de borracha (RPA), da marca Alpha Technologies, de acordo com a norma ${ }^{[7 b]}$ ASTM D 6204.

A análise termo-dinâmico-mecânica foi realizada no analisador DMTA, da marca Rheometric Scientific, modelo MK III, nas seguintes condições: frequiência, $1 \mathrm{~Hz}$; velocidade de aquecimento, $2{ }^{\circ} \mathrm{C} / \mathrm{min}$; modo, flexão; e faixa de temperatura, -80 a $70{ }^{\circ} \mathrm{C}$. Para esse ensaio, os materiais foram prensados a $120{ }^{\circ} \mathrm{C}$, durante $15 \mathrm{~min}$, à pressão de 4MPa, e espessura de $1 \mathrm{~mm}$.

\section{Resultados e Discussão}

A Figura 1 apresenta os resultados de viscosidade e relaxação Mooney das composições estudadas. É importante ressaltar que, para as composições de

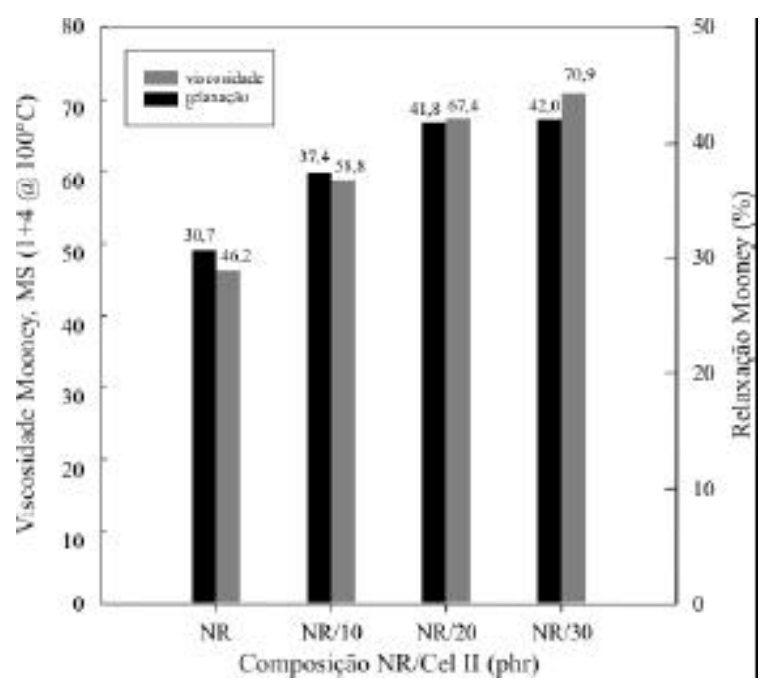

Figura 1 - Viscosidade e relaxação Mooney de composições nãovulcanizadas NR/Cel II 
borracha natural com celulose regenerada, foi utilizado o rotor pequeno, MS, para determinação da viscosidade Mooney, uma vez que os valores medidos com o rotor maior superavam os limites do equipamento para composições com teores de carga acima de 20phr. Pode ser observado que a viscosidade Mooney aumenta muito com a incorporação da carga, conforme esperado. A relaxação Mooney (MR) é definida ${ }^{[8]}$ por:

$$
\mathrm{MR}=\left[\mathrm{M}_{30} / \mathrm{ML}_{(1+4)}\right] \times 100
$$

onde $\mathrm{M}_{30}$ é a viscosidade medida 30s após a parada do rotor, quando o valor $\mathrm{ML}_{(1+4)}$ é determinado.

Para relaxação Mooney os resultados mostram que a presença da celulose regenerada limita a relaxação da borracha, e houve modificação significativa de comportamento com adição da carga celulósica.

Há mais de 60 anos a viscosidade Mooney é usada para a caracterização dos elastômeros crus ou de composições não vulcanizadas na indústria de borracha. Porém, o valor da viscosidade Mooney é obtido à baixa taxa de cisalhamento, e não detecta variações estruturais que afetam o processamento e desempenho final do composto, como é o caso do RPA. Neste equipamento, a distribuição de peso molecular, ramificação, razão entre comonômeros, são alguns exemplos que podem ser detectados ${ }^{[5,9]}$.

A análise termo-dinâmico-mecânica (DMTA) permite a obtenção de vários parâmetros, sendo um deles a relação entre o módulo de perda e o de armazenamento, conhecido como tan delta, cujo valor

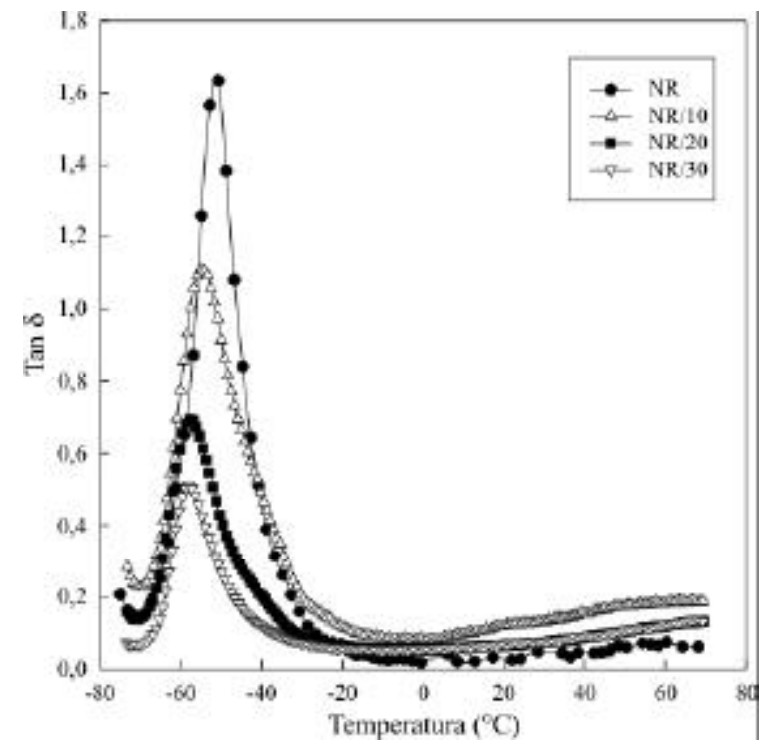

Figura 2. Tan delta versus temperatura de composições não-vulcanizadas $\mathrm{NR} / \mathrm{Cel} \mathrm{II}$ máximo caracteriza a temperatura de transição vítrea, $\mathrm{T}_{\mathrm{g}}$. A variação da tan delta com a temperatura para as composições sob avaliação é mostrada na Figura 2. Pode ser visto que a adição de celulose regenerada diminui a $\mathrm{T}_{\mathrm{g}}$ da borracha natural indicando que, neste caso, a carga promove um afastamento entre as cadeias macromoleculares da borracha, o que acarreta sua maior flexibilidade e, portanto, menor $\mathrm{T}_{\mathrm{g}}$. Além disso houve um grande aumento no módulo elástico das composições com a incorporação da celulose regenerada, o que está relacionado aos valores mais baixos de tan delta, quando comparados ao da composição sem carga. Os picos mais estreitos da tan delta para as composições NR/Cel II indicam boa dispersão da carga celulósica na matriz borrachosa ${ }^{[10,11]}$.

Na Figura 3 é apresentada a variação do torque elástico com a freqüência das composições de borracha natural. É possível verificar o aumento do torque elástico com a adição de celulose regenerada, e que este não varia significativamente para frequiências superiores a 50cpm, em todas as composições estudadas.

A variação do torque viscoso com a freqüência das composições de borracha natural é mostrada na Figura 4. Pode ser observado o aumento deste torque com a adição crescente de carga, e que o mesmo é independente da freqüência, em toda a extensão estudada. Além disso, para as composições NR/Cel II, os valores do torque viscoso são muito menores do que do torque elástico (Figura 3).

A Figura 5 mostra a variação da viscosidade com

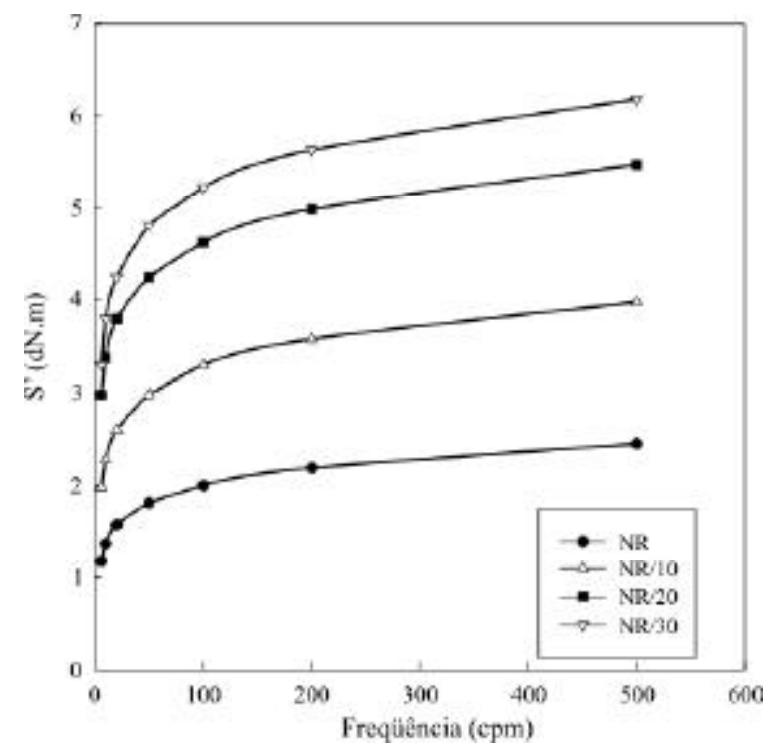

Figura 3. Torque elástico versus freqüência de composições nãovulcanizadas $\mathrm{NR} / \mathrm{Cel}$ II a $100^{\circ} \mathrm{C} ; 0,5^{\circ}$ 


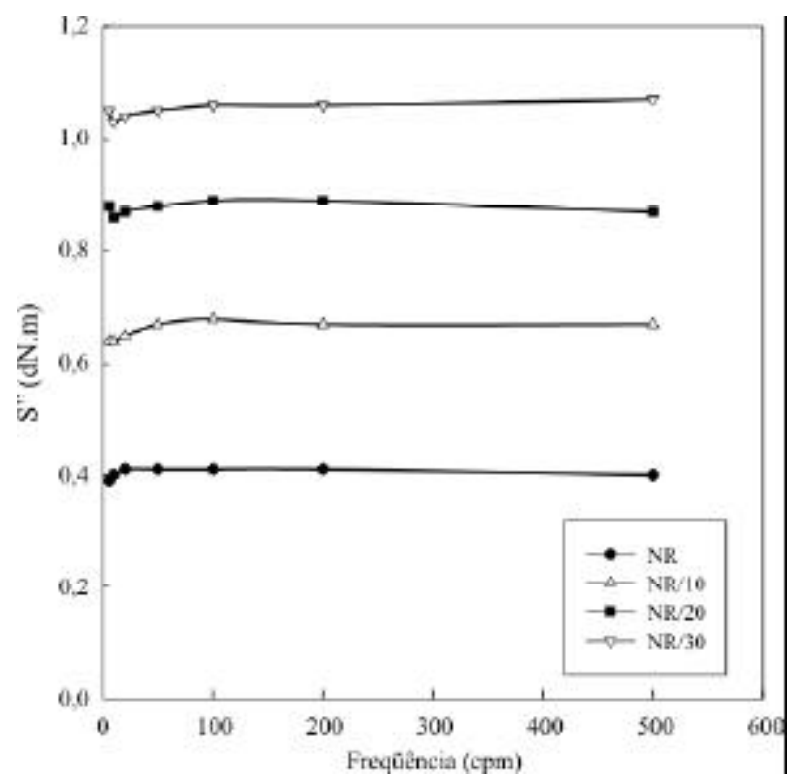

Figura 4. Torque viscoso versus frequiência de composições nãovulcanizadas $\mathrm{NR} / \mathrm{Cel}$ II a $100{ }^{\circ} \mathrm{C} ; 0,5^{\circ}$

a frequiência das composições estudadas. É claramente observada a influência da celulose regenerada pelo grande aumento da viscosidade das composições. Além disso, pode ser verificada a variação inversa da viscosidade com a freqüência, e que ela é independente do teor de carga entre 100 e $500 \mathrm{cpm}$.

As Figuras 3, 4 e 5 mostram a forte influência da celulose regenerada nos parâmetros reológicos das composições estudadas, o que indica boa dispersão desta carga na matriz elastomérica. Além disso, entre 100 e 500cpm, todas as composições com celulose

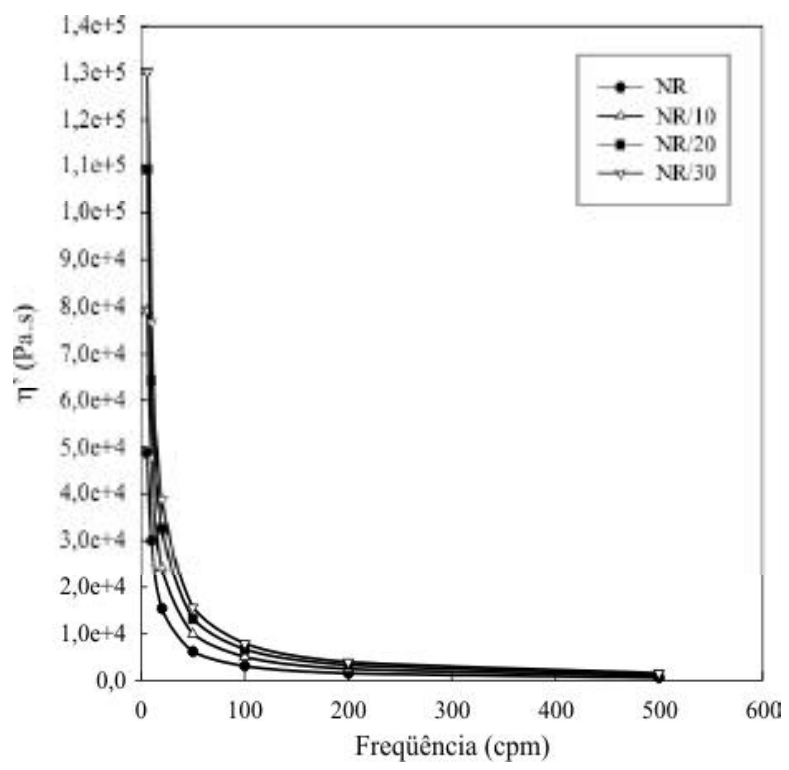

Figura 5. Viscosidade dinâmica real versus freqüência de composições não-vulcanizadas $\mathrm{NR} / \mathrm{Cel}$ II a $100{ }^{\circ} \mathrm{C} ; 0,5^{\circ}$

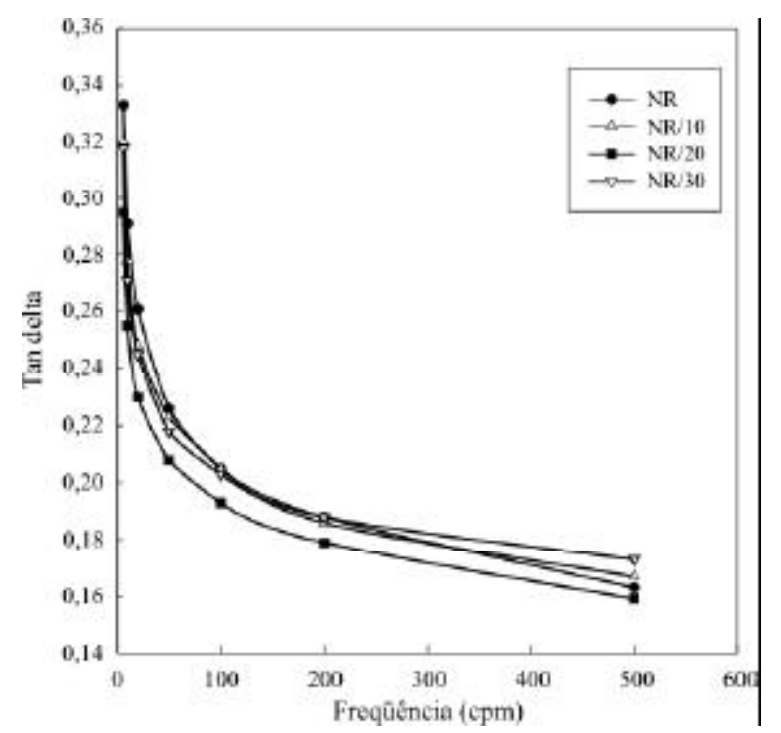

Figura 6. Tan delta versus frequiência de composições não-vulcanizadas $\mathrm{NR} / \mathrm{Cel}$ II a $100{ }^{\circ} \mathrm{C} ; 0,5^{\circ}$

regenerada têm comportamento paralelo ao da borracha natural, o que sugere uma forte interação física entre os componentes, já que a presença da carga celulósica aumenta os valores dos parâmetros reológicos, conforme esperado, sem alterar o perfil do comportamento reológico da borracha natural.

A Figura 6 mostra a variação da tan delta com a freqüência das composições de borracha natural estudadas. Pode ser observado que os valores da tan delta diminuem com o aumento da frequiência, e não há diferenças significativas entre as composições estudadas, na faixa de 100 a $500 \mathrm{cpm}$.

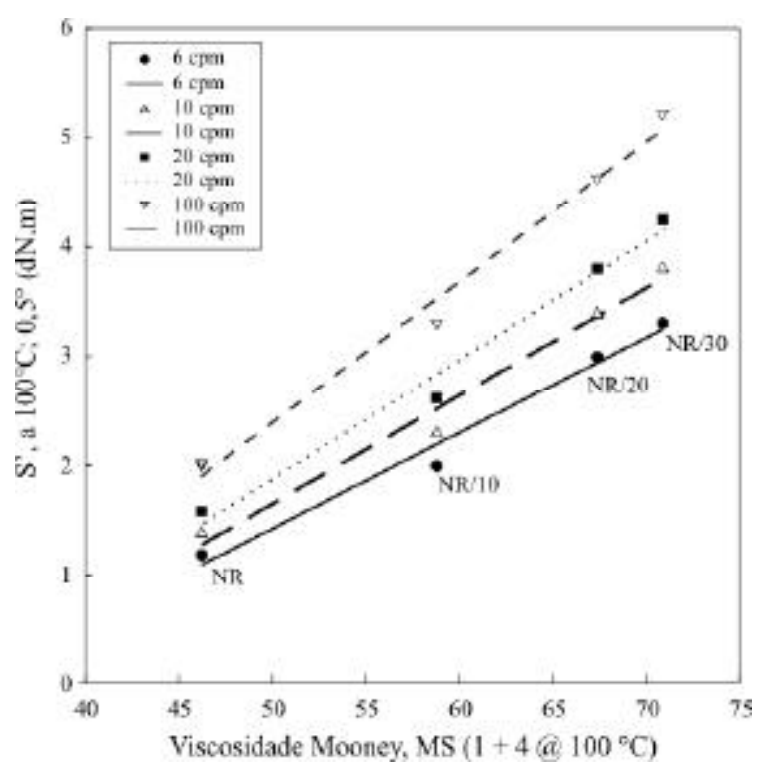

Figura 7. Torque elástico versus viscosidade Mooney de composições não-vulcanizadas NR/Cel II 
A correlação entre torque elástico e viscosidade Mooney das composições de borracha natural é apresentada na Figura 7. É possível verificar uma relação linear entre as duas propriedades, independente da freqüência, nas condições experimentais usadas. Em todos os casos, o coeficiente de correlação encontrado foi de 0,98 para todas as composições estudadas. As composições NR/Cel II apresentam comportamento diferente dos sistemas de borracha natural com negro de fumo, onde o coeficiente de correlação é reduzido pelo aumento da frequiência, passando de 0,98 a $0,89^{[12]}$. Sendo assim, os resultados obtidos para as composições NR/Cel II indicam uma distribuição de carga a nível molecular na matriz elastomérica. Vale ressaltar que as composições NR/Cel II vulcanizadas têm resistência mecânica superior às composições vulcanizadas de borracha natural com negro de fumo ${ }^{[13]}$.

De acordo com a literatura ${ }^{[9]}$, a maioria dos elastômeros sintéticos mostra comportamento viscoelástico linear para deformações inferiores a $14 \%\left(\operatorname{arco}\right.$ de $1^{\circ}$ ). O comportamento reológico na região viscoelástica linear é útil para detectar diferenças entre os polímeros, como distribuição de peso molecular, ramificações, etc; e, na região viscoelástica não-linear, pode ser muito importante quanto ao processamento do material.

Grandes diferenças entre os polímeros têm sido observadas, em altas deformações, quando se usa torque elástico ( $\left.S^{\prime}\right)$ ao invés de módulo elástico $\left(G^{\prime}\right)$. Tipicamente, $S$ ' passa por um máximo com o aumento

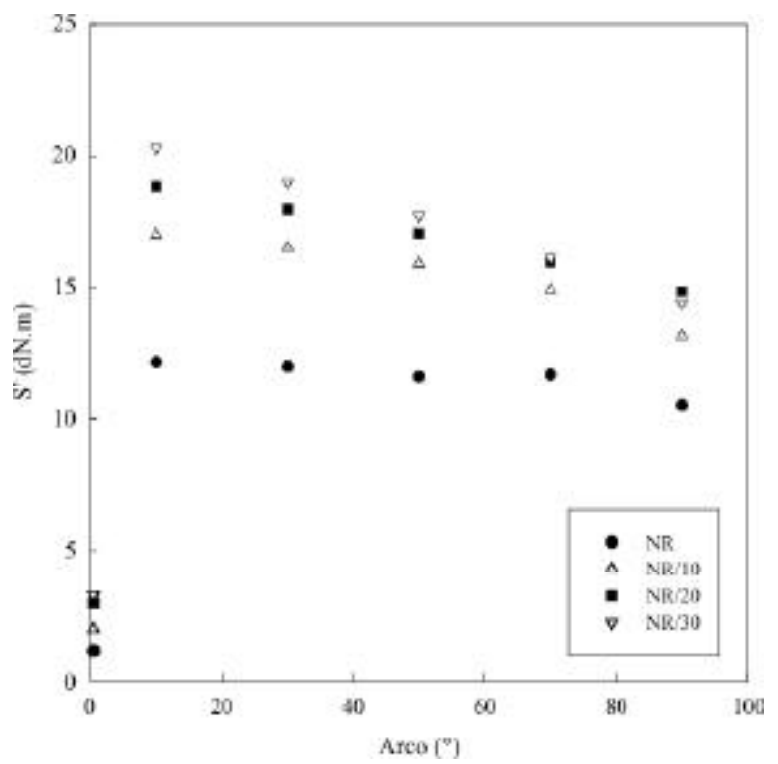

Figura 8. Torque elástico versus arco de composições não-vulcanizadas $\mathrm{NR} / \mathrm{Cel}$ II a $100{ }^{\circ} \mathrm{C} ; 6 \mathrm{cpm}$

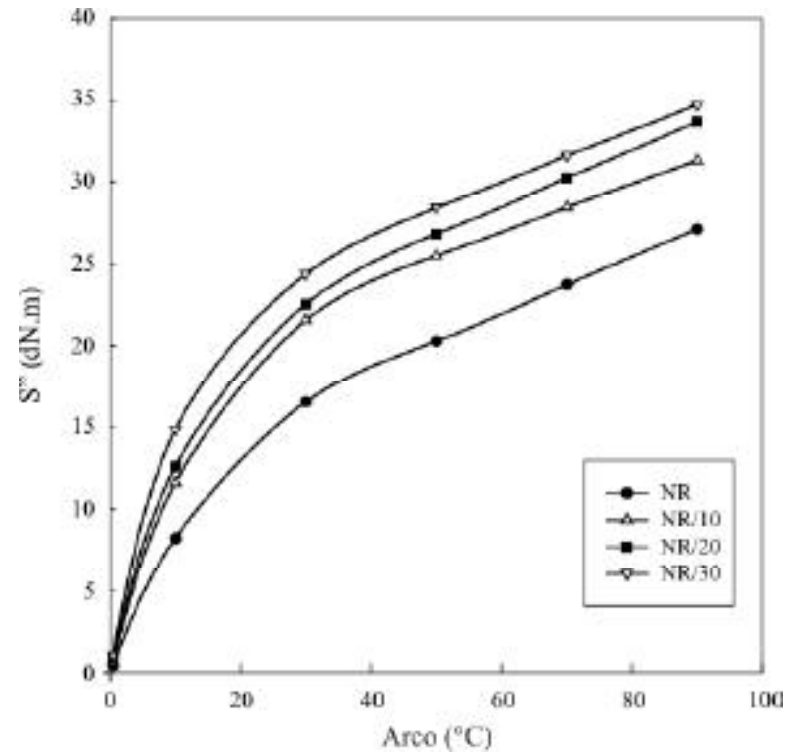

Figura 9. Torque viscoso versus arco de composições não-vulcanizadas $\mathrm{NR} / \mathrm{Cel} \mathrm{II} \mathrm{a} 100{ }^{\circ} \mathrm{C}$; $6 \mathrm{cpm}$

da deformação. Mudanças em S', a altas deformações, estão relacionadas às diferenças de processamento. Assim a resposta de torque, elástico ( $\left.\mathrm{S}^{\prime}\right)$ e viscoso (S"), é possivelmente melhor para o estudo das mudanças reológicas que ocorrem a altas deformações na região viscoelástica não-linear ${ }^{[9]}$.

As Figuras 8 e 9 mostram a resposta dos torques elástico e viscoso com a variação do arco. Pode ser observado o máximo de S' (Figura 8), conforme a literatura. Para as composições NR/Cel II estudadas, todas apresentaram o valor máximo de $\mathrm{S}$ ' para o mesmo valor de arco e, comparando os resultados para a mesma composição, os valores do torque viscoso são muito maiores do que do torque elástico. Em sistemas com negro de fumo, diferenças entre as cargas negras são evidenciadas pelo gráfico S'x arco. Os negros de fumo usados diferem em tipo, teor, e razão carga/óleo. Nesses sistemas o valor máximo de $S^{\prime}$ ocorreu em diferentes valores de $\operatorname{arco}^{[9]}$. Nas Figuras 8 e 9 as composições com celulose regenerada apresentaram comportamento paralelo ao da borracha natural, em toda a faixa estudada.

\section{Conclusões}

O processo de co-coagulação empregado para a obtenção das composições não-vulcanizadas de borracha natural com celulose regenerada promove uma excelente dispersão molecular da carga na matriz borrachosa, o que pôde ser comprovado pelos 
resultados reológicos e dinâmicos apresentados. Além disso, a celulose regenerada influencia fortemente as propriedades viscoelásticas da borracha natural, mas não altera o perfil do seu comportamento reológico.

\section{Agradecimentos}

Os autores agradecem ao Conselho Nacional de Desenvolvimento Científico e Tecnológico (CNPq) pelo apoio financeiro, à Fibra S.A. pela doação do xantato de celulose e à Petroflex Indústria e Comércio S.A. pela realização dos ensaios no RPA e de viscosidade Mooney.

\section{Referências Bibliográficas}

1. Goetler, L. A.; Shen, K. S. - Rubber Chem. Technol., 56, p.619 (1983).

2. M. Leon, Brit. 444,363 (1936).

3. Seymour, R. B. - Revista de Plásticos Modernos, 306, p.679 (1981).

4. Bagley, E. B.; Dixon, R. E. - Trans. Soc. Rheol., 18, p.371 (1974).

5. Dick, J. S.; Pawlowski, H. - Rubber World, January, p.21 (1997).
6. Rohn, C. L. - "Rheological Testing", in: Analytical Polymer Rheology - Structure-Processing-Property Relationships, cap.12, Hanser Publishers, New York (1995).

7. American Society for Testing and Materials

a) ASTM D 1646-98a - Rubber- viscosity, stress relaxation, and pre-vulcanization characteristics (Mooney viscometer)

b) ASTM D 6204-99 - Rubber- measurement of unvulcanized rheological properties using rotorless shear rheometers

8. Leblanc, J.L. - "Rhéologie des élastomères et mise en ouevre des polymères”, Ed. Artel, Belgique, (1996).

9. Dick, J.S.; Pawlowski, H. - Rubber World, January, p.20 (1995).

10. Medalia, A.I. - Rubber Chem. Technol., 51, p.437 (1978).

11. Sombatsompop, N. - Polym.-Plast. Technol. Eng., 37, p.333 (1998).

12. Dick, J.S.; Harmon, C.; Vare, A. Polym. Testing, 18, p.327 (1999).

13. Martins, A.F.; Visconte, L.L.Y.; Nunes, R.C.R. Kautsch. Gummi Kunstst., in press

Recebido: 10/08/01

Aprovado: 30/08/02 